\title{
Lay Health Coaching to Increase Appropriate Inhaler Use in COPD: A Randomized Controlled Trial
}

Rachel Willard-Grace, MPH

Chris Chirinos, $B A^{1}$

Jessica Wolf, BS ${ }^{1}$

Denise DeVore, $B S^{1}$

Beatrice Huang, $B A^{1}$

Danielle Hessler, $\mathrm{PbD}^{1}$

Stephanie Tsao, MSN ${ }^{2}$

George Su, MD ${ }^{3}$

David H. Thom, MD, $P b D^{4}$

'Department of Family and Community Medicine, University of California San Francisco, San Francisco, California

${ }^{2}$ San Francisco Department of Public Health, San Francisco, California

${ }^{3}$ Department of Medicine: Pulmonology, Critical Care, Allergy and Sleep Medicine Program, University of California San Francisco, San Francisco, California

${ }^{4}$ Department of Medicine, Stanford University School of Medicine, Palo Alto, California

Conflicts of interest: authors report none.

\section{CORRESPONDING AUTHOR}

Rachel Willard-Grace, MPH 1001 Potrero Ave, Ward 83

San Francisco, CA 94110

Rachel.Willard@ucsf.edu

\begin{abstract}
PURPOSE Poor adherence to medications is more prevalent for chronic obstructive pulmonary disease (COPD) than for other chronic conditions and is associated with unfavorable health outcomes. Few interventions have successfully improved adherence for COPD medications; none of these use unlicensed health care personnel. We explored the efficacy of lay health coaches to improve inhaler adherence and technique.
\end{abstract}

METHODS Within a randomized controlled trial, we recruited English- and Spanish-speaking patients with moderate to severe COPD from urban, public primary care clinics serving a low-income, predominantly African American population. Participants were randomized to receive 9 months of health coaching or usual care. Outcome measures included self-reported adherence to inhaled controller medications in the past 7 days and observed technique for all inhalers. We used generalized linear models, controlling for baseline values and clustering by site.

RESULTS Baseline adherence and inhaler technique were uniformly poor and did not differ by study arm. At 9 months, health-coached patients reported a greater number of days of adherence compared with usual care patients (6.4 vs 5.5 days; adjusted $P=.02$ ) and were more likely to have used their controller inhalers as prescribed for 5 of the last 7 days (90\% vs 69\%; adjusted $P=.008)$. They were more than 3 times as likely to demonstrate perfect technique for all inhaler devices (24\% vs $7 \%$; adjusted $P=.01$ ) and mastery of essential steps ( $40 \%$ vs $11 \%$; adjusted $P<.001)$.

CONCLUSIONS Health coaching may provide a scalable model that can improve care for people living with COPD.

Ann Fam Med 2020;18:5-14. https://doi.org/10.1370/afm.2461.

\section{INTRODUCTION}

hronic obstructive pulmonary disease (COPD) affects more than 14 million US adults ${ }^{1}$ and is a leading cause of 30-day hospital readmissions in the United States. ${ }^{2}$ Use of inhaled medications is recommended by the Global Initiative for Chronic Obstructive Lung Disease (GOLD) international guidelines ${ }^{3}$ and is associated with reduced exacerbations, decreased hospitalizations, ${ }^{4,5}$ fewer symptoms ${ }_{,}^{6}$ better disease control, and longer survival., ${ }^{5,7}$ Adherence to medications is worse for COPD than for most other conditions, however, ${ }^{8}$ with multiple large, claims-based studies estimating adherence rates of $23 \%$ to $43 \%{ }^{9-11}$ Contributors to poor adherence for inhaled COPD medications include complex medication regimens, ${ }_{1}^{12}$ polypharmacy, ${ }_{1}^{13}$ poor understanding of the disease and how inhalers relieve symptoms, ${ }^{14,15}$ confusion about prescribed regimens, ${ }^{16}$ high costs of inhalers, current smoking, poor clinic attendance, comorbid illness, ${ }^{17-20}$ depression, ${ }^{21,22}$ and poor trust in the clinician. ${ }^{17,19}$

Even for patients using their medications, poor inhaler technique can result in underdosing. ${ }^{23}$ More than $70 \%$ of patients use their inhalers incorrectly, and this rate has remained unchanged in 40 years. ${ }^{24}$ Barriers to effective inhaler use include the complexity and diversity of devices, which require correct execution of 6 to 8 steps that may be contradic- 
tory across device types..$^{25}$ Moreover, clinicians often lack knowledge of how to correctly use the devices, with two-thirds unable to demonstrate critical steps of inhaler use. ${ }^{26,27}$

Poor adherence and errors in technique are even more pronounced for low-income and minority patients and those with lower educational attainment. ${ }^{28-32}$ These factors may contribute to greater disease severity, poorer disease-related quality of life, more hospitalizations, and a greater risk of dying from COPD. ${ }^{33,34}$

Multiple recent reviews have found a dearth of studies of interventions that improved adherence for COPD. ${ }^{35-37}$ The few interventions to successfully improve medication adherence or inhaler technique for COPD relied on pharmacists or nurses. ${ }^{32,36}$

Health coaching is a patient-centered, team-based model of care. Health coaches facilitate shared decision making ${ }^{38}$ and equip patients with the knowledge, skills, and confidence to manage their conditions. ${ }^{39,40}$ Although health coaching for COPD has been delivered with some success by nurses and respiratory therapists, ${ }^{41-43}$ these resources are rarely available in the context of primary care, particularly in resourcelimited settings. Lay health coaches have been efficacious at improving medication adherence and disease control for other conditions. ${ }^{44-46}$

The Aides in Respiration (AIR) health-coaching study sought to improve disease-related quality of life, reduce exacerbations, and increase exercise capacity for people living with COPD. As previously published, improvements in these primary outcomes did not reach statistical significance. ${ }^{47}$ In this article, we report on secondary outcomes related to inhaler adherence and technique. We explored the efficacy of health coaching using trained, unlicensed personnel to improve adherence to and technique in using inhalers among a lowincome, predominantly African American population living with moderate to severe COPD.

\section{METHODS}

The AIR health-coaching study was a multisite, singleblinded randomized controlled trial. The study protocol was approved by the UCSF Human Research Protection Program (approval no. 14-12872) and registered with clinicaltrials.gov (NCT02234284). The study protocol ${ }^{48}$ and primary outcomes ${ }^{47}$ have been previously published.

\section{Setting}

This study was conducted at 7 urban, county-operated primary care clinics, including 2 academic residency teaching practices, that primarily serve a low-income, publicly insured patient population. Pulmonary spe- cialty care was available through the public hospital that was part of the health network and could be accessed via an electronic consultation system.

\section{Participants}

Enrollees were English- or Spanish-speaking patients at least 40 years old who were contactable by telephone and planned to continue to receive care at 1 of the 7 study sites. Clinical eligibility included having COPD, confirmed by a post-bronchodilator spirometry ratio of forced expiratory volume in 1 second $\left(\mathrm{FEV}_{1}\right)$ to forced vital capacity (FVC) of less than 0.70 , or by review by a pulmonologist, that was moderate to severe as defined in published study protocol. ${ }^{48}$ Health coaches for this study held bachelor's degrees from 4-year colleges but were not licensed health care professionals. Both health coaches were fluent in English and Spanish.

\section{Identification and Recruitment}

Potential recruits were identified from targeted diagnoses in billing records or hospital census data, as well as referrals from clinicians at specialty and primary care sites. Medical chart review and primary clinician review were conducted to determine eligibility based on clinical criteria. Research assistants (RAs) contacted potentially eligible patients by telephone using a recruitment script or by letter.

\section{Enrollment and Randomization}

RAs met with eligible patients to secure consent, verbally administered a questionnaire, and observed inhaler techniques. Participants received up to $\$ 30$ at baseline and $\$ 60$ at 9 months in acknowledgment of their study participation. A random binary sequence, created by the project manager and stratified by site, was used to order study arm assignment into sequentially numbered envelopes in a 1:1 ratio. Once baseline measures were complete, the RA asked the patient to open a sealed envelope with a randomization card indicating assignment to usual care or health coaching. Study investigators and the data safety monitoring board were blinded to assignment until analyses were finalized.

\section{Health-Coaching Intervention}

Health coaches received more than 100 hours of training using a health-coaching curriculum ${ }^{49}$ supplemented by COPD-specific content. The curriculum covered active listening and nonjudgmental communication, navigating health care systems, creating selfmanagement goals, and use of teach-back (closing the loop) methods. ${ }^{50}$ COPD-specific training delivered by pulmonary specialists included inhaled medications. 
Health coaches worked with patients for 9 months, with a maximum caseload of 30 patients at any given time. They accompanied patients to visits with primary care clinicians, pulmonary clinicians, or both, met with them individually in the community or at their home and conducted telephone calls between in-person visits. Health coaches addressed barriers to medication adherence and conducted teach-back to improve inhaler technique. The minimum frequency of contact was once every 3 weeks. Patient interactions were documented in a database created for the study, including date, time, topics discussed, and relevant notes. Health coaches met with a supervising pulmonary specialist nurse practitioner at least weekly.

\section{Usual Care}

Patients randomized to usual care received any resources provided by their clinic as part of standard care. These resources included but were not limited to visits with their primary care clinician, pulmonary clinician, or both; COPD education classes; pulmonary rehabilitation; and smoking cessation resources.

\section{Measures}

On enrollment and at 9 months, RAs reviewed each inhaled medication presented by the patient. They asked patients to demonstrate how they used their inhalers, and recorded inhaler technique using a standardized checklist to mark successful completion of each step of inhaler use. Checklists were adapted from those developed by Melani and colleagues ${ }^{51,52}$ and modified by the pulmonary specialist members of the team to match standard inhaler education instructions for the most commonly used device types, including Diskus (GSK), HandiHaler (Boehringer Ingelheim), Respimat (Boehringer Ingelheim), and variations for metered dose inhalers for open- and closed-mouth techniques and use of a spacer. Patients using more than 1 type of device were asked to demonstrate use of each device. Patients used their own inhalers when available; demonstration inhalers were available for patients who did not bring their own inhalers. Medication adherence was assessed by reading the dosing instructions and asking, "In the past 7 days, how many days did you take this medicine exactly as it was prescribed?"44

\section{Outcomes}

Perfect inhaler use was defined as successful completion of every step of inhaler use for every inhaler for which use was demonstrated. In addition, the study investigators identified a priori the essential steps required for delivery of medication and created a dichotomous variable for adequate use, defined as suc- cessful completion of every essential step required for medication delivery, for every inhaler for which use was demonstrated. Finally, a continuous, weighted score was created by assigning 2 points for each essential step and 1 point for each additional step completed successfully. Weighted scores for each inhaler were normalized on a 100-point scale, and a mean was generated across inhalers demonstrated by each patient.

Medication adherence was analyzed, per a previously published protocol ${ }_{,}^{44}$ both as a continuous variable (the mean number of days of medication adherence in the past 7 days across all controller inhaled medications) and as 2 dichotomous variables: perfect adherence, defined as having taken every controller inhaler as prescribed every day for the past 7 days, and good adherence, defined as having taken controller inhalers as prescribed for an average of at least 5 of the past 7 days.

\section{Quality Assurance}

Before study launch, the project manager conducted skills checks with each RA to ensure that she consistently captured correct information about technique through the inhaler checklist. Spot checks were conducted periodically thereafter to ensure compliance to the study protocol.

\section{Statistical Analyses}

Analysis of inhaler technique was restricted to patients using at least 1 inhaler at baseline, and analysis of medication adherence to patients using at least 1 controller inhaler at baseline. Baseline patient characteristics were compared between study arms and tested for significance using the $\chi^{2}$ test for categorical variables, $t$ tests for normally distributed continuous variables, and nonparametric tests for non-normally distributed continuous variables. Outcomes were compared by assignment arm (intention-to-treat approach) using generalized linear models with a normal distribution, with identity link for continuous outcomes and binomial distribution with logit link for binary outcomes. Hypothesis tests were 2 -sided with $P$ values $<.05$ considered statistically significant. A robust standard error was used to account for clustering and accommodate missing data under the assumption that the outcomes were missing at random. ${ }^{53-57}$ In all models, the baseline level of the outcome was included as a predictor and the follow-up level as the dependent variable.

We tested for differential attrition between study arms with logistic regression models for a preidentified set of variables (age, sex, disease severity, smoking status, substance abuse, and homelessness) with participation at 9 months as the dependent variable and independent variables including study arm, the 


\section{Figure 1. Consolidated standards of reporting trials diagram.}

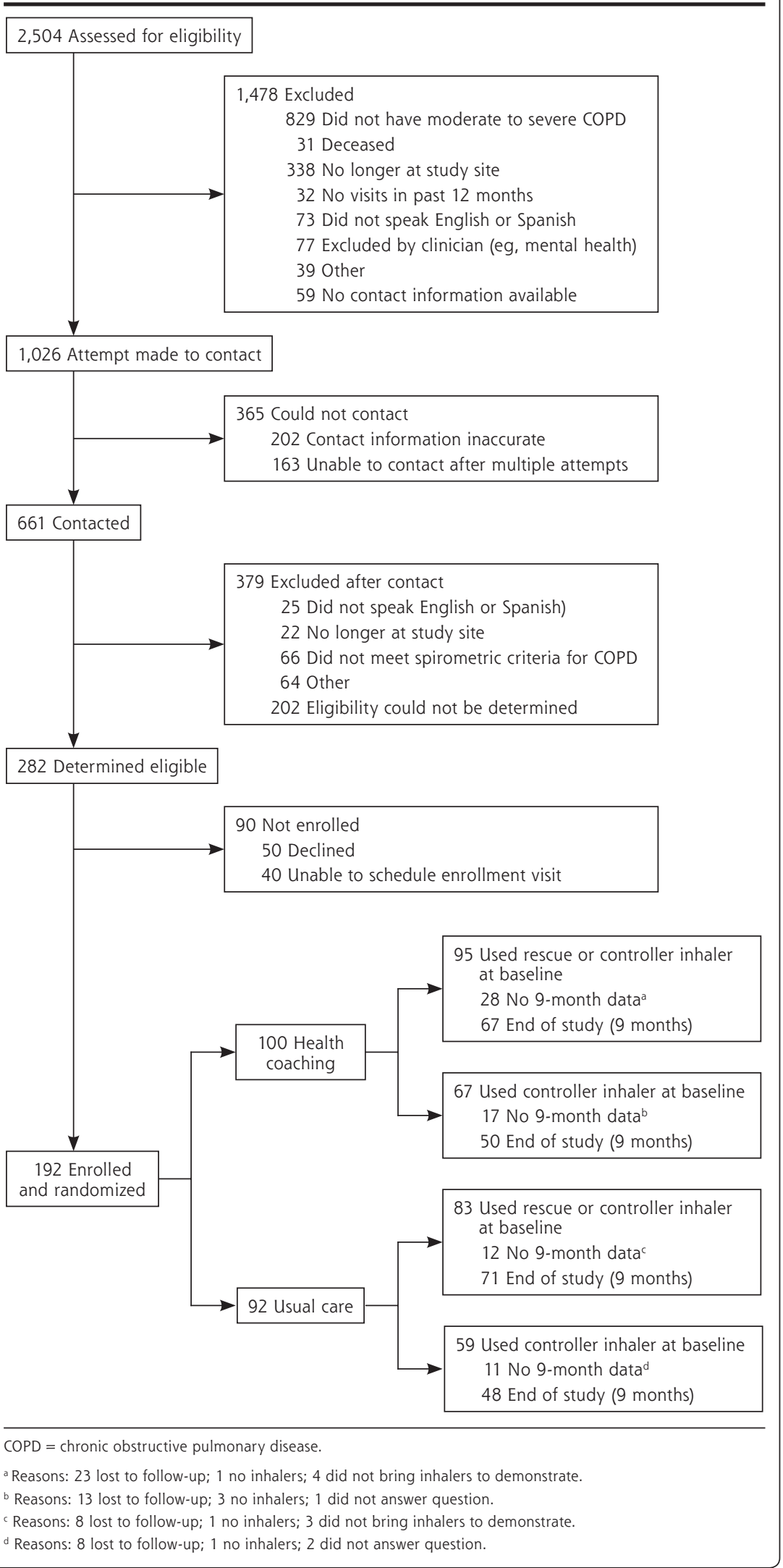

variable, and an interaction term of study arm by the variable. We conducted subgroup analysis for adherence to long-acting muscarinic antagonists (LAMAs). Two planned sensitivity analyses were conducted. First, we imputed missing values in a subset of data using variables from the current analysis, examining 10 iterations and aggregated results; second, we adjusted for season of enrollment, patient age, race, and sex as well as baseline variables that differed between study arms at $P<.10$. Statistical analyses were run using Stata 13.0 (StataCorp, LLC) and multiple imputation using the SPSS Multiple Imputation Procedure (IBM).

\section{RESULTS}

Of 282 patients identified as eligible for the study, 192 (68\%) were enrolled and randomized to receive health coaching $(\mathrm{n}=100)$ or usual care $(\mathrm{n}=92)$ (Figure 1). Of the 178 using a rescue or controller inhaler at baseline, $138(78 \%)$ provided data at 9 months; of the 126 using a controller inhaler, 98 (78\%) provided data at 9 months. Loss to follow-up was greater for the health-coaching arm than for the usual-care arm for the subsample included in the inhaler technique analysis $(29 \%$ vs $14 \% ; P=.02)$. There were no significant differences between the study arms in baseline demographics or disease severity for patients who dropped out of the study. None of the patients participating in the study used oral medications for their COPD.

Participating patients had a mean age of 61 years, and the majority $(66 \%)$ were male (Table 1). More than one-half (57\%) were African American and about one-fifth (17\%) reported Hispanic ethnicity. 
Table 1. Participant Characteristics, by Study Arm ( $=192)$

\begin{tabular}{|c|c|c|c|c|}
\hline \multirow[b]{2}{*}{ Characteristic } & \multirow[b]{2}{*}{$\begin{array}{c}\text { All } \\
(\mathrm{N}=192)\end{array}$} & \multicolumn{3}{|c|}{ Arm } \\
\hline & & $\begin{array}{l}\text { Health-Coaching Arm } \\
\quad(n=100)\end{array}$ & $\begin{array}{l}\text { Usual-Care Arm } \\
\quad(n=92)\end{array}$ & $\begin{array}{c}P \\
\text { Value }\end{array}$ \\
\hline \multicolumn{5}{|l|}{ Demographics } \\
\hline Age, mean (SD), y & $61.3(7.6)$ & $60.7(8.0)$ & $61.9(7.2)$ & NS \\
\hline Male, \% (No.) & $65.5(126)$ & $67.0(67)$ & $64.1(59)$ & NS \\
\hline Works full/part-time outside the home, \% (No.) & $17.8(34)$ & $16.0(16)$ & $19.8(18)$ & NS \\
\hline Income $<\$ 10,000 / y$, \% (No.) & $45.7(84)$ & $45.8(44)$ & $45.5(40)$ & NS \\
\hline Black/African American race, \% (No.) & $56.8(109)$ & $53.0(53)$ & $60.9(56)$ & NS \\
\hline Hispanic/Latino ethnicity, \% (No.) & $16.7(32)$ & $13.0(13)$ & $20.7(19)$ & NS \\
\hline Preferred language is not English, \% (No.) & $12.6(24)$ & $7.0(7)$ & $18.7(17)$ & .02 \\
\hline Education less than high school, \% (No.) & $31.9(61)$ & $27.0(27)$ & $37.4(34)$ & NS \\
\hline $\begin{array}{l}\text { Had visit with pulmonary specialist in } 12 \text { months } \\
\text { before enrollment, \% (No.) }\end{array}$ & $31.3(60)$ & $34.0(34)$ & $28.3(26)$ & NS \\
\hline \multicolumn{5}{|l|}{ Severity of COPD } \\
\hline GOLD classification, \% (No.) ${ }^{a}$ & & & & NS \\
\hline Gold A (low symptoms, low risk) & $4.2(8)$ & $5.1(5)$ & $3.3(3)$ & \\
\hline Gold B (high symptoms, low risk) & $46.3(88)$ & $46.5(46)$ & $46.2(42)$ & \\
\hline Gold C (low symptoms, high risk) & $3.2(6)$ & $4.0(4)$ & $2.2(2)$ & \\
\hline Gold D (high symptoms, high risk) & $46.3(88)$ & $44.4(44)$ & $48.4(44)$ & \\
\hline $\mathrm{FEV}_{1} \%$ of predicted, mean (SD) & $58(20)$ & $55(19)$ & $60(20)$ & NS \\
\hline High COPD symptom score: CAT $\geq 10, \%$ (No.) & $92.7(177)$ & $90.9(90)$ & $94.6(87)$ & NS \\
\hline Ever smoked, \% (No.) & $96.3(184)$ & $99.0(99)$ & $93.4(85)$ & .04 \\
\hline Current smoker, \% (No.) & $53.8(99)$ & $54.6(54)$ & $52.9(45)$ & NS \\
\hline Asthma diagnosis, \% (No.) & $27.6(53)$ & $29.0(29)$ & $26.1(24)$ & NS \\
\hline \multicolumn{5}{|l|}{ Inhaled medications } \\
\hline Uses any inhaler (rescue or controller), \% (No.) & $92.7(178)$ & $95.0(95)$ & $90.2(83)$ & NS \\
\hline Uses controller inhaler, \% (No.) & $65.6(126)$ & $67.0(67)$ & $64.1(59)$ & NS \\
\hline Number of controller inhalers prescribed, mean (SD) & $1.4(0.9)$ & $1.5(0.9)$ & $1.3(0.9)$ & NS \\
\hline \multicolumn{5}{|l|}{ Types of medications prescribed ${ }^{b}$} \\
\hline Short-acting $\beta$ agonist (SABA), \% (No.) & $88.5(170)$ & $90.0(90)$ & $87.0(80)$ & NS \\
\hline Short-acting anticholinergic, \% (No.) & $34.4(66)$ & $32.0(32)$ & $37.0(34)$ & NS \\
\hline Long-acting $\beta$ agonist (LABA), \% (No.) & $56.8(109)$ & $57.0(57)$ & $56.5(52)$ & NS \\
\hline Long-acting muscarinic antagonist (LAMA), \% (No.) & $51.6(99)$ & $59.0(59)$ & $43.5(40)$ & .03 \\
\hline Inhaled corticosteroid (ICS), \% (No.) & $76.0(146)$ & $76.0(76)$ & $76.1(70)$ & NS \\
\hline Correctly identified rescue inhaler, \% (No.) & $88.5(161)$ & $90.7(88)$ & $85.9(73)$ & NS \\
\hline Number of uses of rescue inhaler/day, mean (SD) & $2.5(1.9)$ & $2.7(2.0)$ & $2.3(1.8)$ & NS \\
\hline \multicolumn{5}{|l|}{ Inhaler use } \\
\hline Metered dose inhaler, \% (No.) & $91.8(168)$ & $90.7(88)$ & $93.0(80)$ & NS \\
\hline Closed-mouth technique & $66.1(111)$ & $63.6(56)$ & $68.8(55)$ & NS \\
\hline Open-mouth technique & $8.9(15)$ & $10.2(9)$ & $7.5(6)$ & NS \\
\hline Use with a spacer & $25.0(42)$ & $26.1(23)$ & $23.8(19)$ & NS \\
\hline HandiHaler, \% (No.) & $40.2(70)$ & $42.9(39)$ & $37.4(31)$ & NS \\
\hline Diskus, \% (No.) & $19.4(35)$ & $13.7(13)$ & $25.9(22)$ & .04 \\
\hline Respimat, \% (No.) & $12.0(22)$ & $14.4(14)$ & $9.3(8)$ & $\begin{array}{l}\text { NS } \\
\text { continues }\end{array}$ \\
\hline \multicolumn{5}{|c|}{$\begin{array}{l}\mathrm{COPD}=\text { chronic obstructive pulmonary disease; } \mathrm{CAT}=\mathrm{COPD} \text { Assessment Test; } \mathrm{FEV}_{1}=\text { forced expiratory volume in } 1 \text { second; GOLD }=\text { Global Initiative for Chronic } \\
\text { Obstructive Lung Disease; NS = not significant. }\end{array}$} \\
\hline $\begin{array}{l}\text { a According to the } 2014 \text { GOLD guidelines. } .^{58} \\
\text { b Either alone or in combination with another inhaled medicati }\end{array}$ & & & & \\
\hline
\end{tabular}

Thirty-two percent had less than a high school education, and $37 \%$ reported needing at last some help with health information (not shown). Participants were primarily publicly insured (56\% MediCal and 35\% Medi- care). Thirteen percent were affected by homelessness or housing insecurity. Most (93\%) reported high levels of COPD symptoms. Patients were prescribed a mean of 1.4 controller inhaler medications. 
Table 1. Participant Characteristics, by Study Arm (N = 192) (continued)

\begin{tabular}{|c|c|c|c|c|}
\hline \multirow[b]{2}{*}{ Characteristic } & \multirow[b]{2}{*}{$\begin{array}{c}\text { All } \\
(\mathrm{N}=192)\end{array}$} & \multicolumn{3}{|c|}{ Arm } \\
\hline & & $\begin{array}{l}\text { Health-Coaching Arm } \\
\qquad(n=100)\end{array}$ & $\begin{array}{l}\text { Usual-Care Arm } \\
\quad(n=92)\end{array}$ & $\begin{array}{c}P \\
\text { Value }\end{array}$ \\
\hline $\begin{array}{l}\text { Number days (of last 7) patient reports having taken } \\
\text { medications as prescribed, mean (SD) }\end{array}$ & $5.6(2.3)$ & $5.7(2.1)$ & $5.4(2.4)$ & NS \\
\hline Perfect adherence, \% (No.) ${ }^{c}$ & $59.5(75)$ & $64.2(43)$ & $54.2(32)$ & NS \\
\hline Good adherence, \% (No.) ${ }^{d}$ & $77.0(97)$ & $77.6(52)$ & $76.3(45)$ & NS \\
\hline \multicolumn{5}{|l|}{ Inhaler use technique } \\
\hline Perfect use of all inhalers, \% (No.) ${ }^{e}$ & $2.8(5)$ & $4.2(4)$ & $1.2(1)$ & NS \\
\hline Adequate use of all inhalers, $\%(\mathrm{No} .)^{f}$ & $9.6(17)$ & $12.6(12)$ & $6.0(5)$ & NS \\
\hline Weighted inhaler technique score, mean $(S D)^{g}$ & $72.3(16.2)$ & $72.9(16.3)$ & $71.5(16.1)$ & NS \\
\hline \multicolumn{5}{|c|}{$\begin{array}{l}\text { COPD = chronic obstructive pulmonary disease; } C A T=\text { COPD Assessment Test; FEV } \text { F }_{1} \text { forced expiratory volume in } 1 \text { second; GOLD = Global Initiative for Chronic } \\
\text { Obstructive Lung Disease; NS = not significant. }\end{array}$} \\
\hline $\begin{array}{l}\text { a According to the } 2014 \text { GOLD guidelines }{ }^{58} \\
\text { b Either alone or in combination with another inhaled medicat } \\
\text { c Report of taking all medications as prescribed in the last } 7 d \\
\text { d Report of taking all medications as prescribed for at least } 5 \\
\text { e Successful completion of every step of inhaler use for every } \\
\text { i Successful completion of every essential step required for me } \\
{ }^{9} \text { On a scale of } 0 \text { to } 100 \text {, where higher score indicates better te }\end{array}$ & $\begin{array}{l}\text { ast } 7 \text { days. } \\
\text { for which use wa } \\
\text { nelivery, for ev }\end{array}$ & $\begin{array}{l}\text { onstrated. } \\
\text { naler for which use was demor }\end{array}$ & ated. & \\
\hline Note: Data missing for some patients for some characteristics. & & & & \\
\hline
\end{tabular}

Table 2. Adherence to Controller Inhalers at Baseline and 9 Months, by Study Arm $(N=98)$

\begin{tabular}{|c|c|c|c|c|c|c|c|}
\hline \multirow[b]{2}{*}{ Measure } & \multicolumn{2}{|c|}{$\begin{array}{l}\text { Health-Coaching } \\
\begin{array}{c}\text { Arm } \\
(n=50)\end{array}\end{array}$} & \multicolumn{2}{|c|}{$\begin{array}{l}\text { Usual-Care } \\
\text { Arm } \\
(n=48)\end{array}$} & \multirow{2}{*}{$\begin{array}{c}\text { Difference, } \\
\%\end{array}$} & \multirow{2}{*}{$\begin{array}{l}\text { Adjusted } \\
\text { Difference, \% } \\
(95 \% \mathrm{Cl})^{\mathrm{a}}\end{array}$} & \multirow{2}{*}{$\begin{array}{c}P \\
\text { Value }\end{array}$} \\
\hline & Baseline & 9 Months & Baseline & 9 Months & & & \\
\hline $\begin{array}{l}\text { Number days (of the last 7) } \\
\text { patient reported taking } \\
\text { medications as prescribed, } \\
\text { mean (SD) }\end{array}$ & $5.9(2.0)$ & $6.4(1.3)$ & $5.6(2.4)$ & $5.5(2.0)$ & 0.84 & 0.75 (0.13 to 1.37$)$ & .02 \\
\hline Perfect adherence, \% (No.) & $66.0(33)$ & $70.0(35)$ & $58.3(28)$ & $54.2(26)$ & 15.8 & $15.2(-4.3$ to 34.8$)$ & .13 \\
\hline Good adherence, \% (No.) & $84.0(42)$ & $90.0(45)$ & $79.2(38)$ & $68.8(33)$ & 21.2 & 26.4 (6.9 to 49.9$)$ & .008 \\
\hline
\end{tabular}

At baseline, the usual care arm had a higher proportion of patients whose preferred language was not English (19\% vs 7\%), and the health-coaching arm had more patients with a history of smoking (99\% vs 93\%) (Table 1). Patients in the health-coaching arm were more likely to have been prescribed a LAMA (59\% vs $44 \%)$ and less likely to use a Diskus device ( $14 \%$ vs $26 \%)$ at baseline. Baseline adherence to controller inhalers and correct inhaler use did not differ by study arm.

At 9 months, patients in the health-coaching arm reported a significantly greater number of days of adherence to controller inhalers compared with counterparts in the usual-care arm (6.4 vs 5.5 days; Table 2) and were more likely to have taken all of their medications as prescribed for 5 of the last 7 days $(90 \%$ vs $69 \%$ ). Patients receiving health coaching were 3 times as likely to demonstrate perfect technique of all inhaler devices ( $24 \%$ vs $7 \%$ ), and their weighted inhaler technique score was about 11 points higher (Table 3). When considering the essential steps of inhaler use required for medication delivery, $40 \%$ of health-coached patients vs $11 \%$ of usual-care patients could demonstrate adequate use at 9 months.

The same pattern of results persisted when using imputed values or adjusting for season of enrollment; patient age, race, and sex; and baseline variables that differed between study arms. A subgroup analysis for LAMAs yielded similar results (not shown).

\section{DISCUSSION}

\section{Key Findings}

To our knowledge, this is the first study to demonstrate that unlicensed, trained health coaches may 
Table 3. Inhaler Technique for Controller and Rescue Medications at Baseline and 9 Months, by Study Arm ( $\mathrm{N}=138)$

\begin{tabular}{|c|c|c|c|c|c|c|c|}
\hline \multirow[b]{2}{*}{ Measure } & \multicolumn{2}{|c|}{$\begin{array}{l}\text { Health-Coaching } \\
\text { Arm } \\
(n=67)\end{array}$} & \multicolumn{2}{|c|}{$\begin{array}{c}\text { Usual-Care } \\
\text { Arm } \\
(n=71)\end{array}$} & \multirow{2}{*}{$\begin{array}{c}\text { Difference, } \\
\%\end{array}$} & \multirow{2}{*}{$\begin{array}{c}\text { Adjusted } \\
\text { Difference, \% } \\
(95 \% \mathrm{Cl})^{\mathrm{a}}\end{array}$} & \multirow{2}{*}{$\begin{array}{c}P \\
\text { Value }\end{array}$} \\
\hline & Baseline & 9 Months & Baseline & 9 Months & & & \\
\hline $\begin{array}{l}\text { Perfect use of all inhalers, } \\
\% \text { (No.) }\end{array}$ & $4.5(3)$ & $23.9(16)$ & $1.4(1)$ & $7.0(5)$ & 16.9 & $24.8(4.2-42.6)$ & .01 \\
\hline $\begin{array}{l}\text { Adequate use of all inhalers, } \\
\% \text { (No.) }\end{array}$ & $9.0(6)$ & $40.3(27)$ & $5.6(4)$ & $11.3(8)$ & 29.0 & $40.0(20.7-59.2)$ & $<.001$ \\
\hline $\begin{array}{l}\text { Weighted inhaler technique } \\
\text { score, mean (SD) }\end{array}$ & $71.9(15.7)$ & $88.9(10.2)$ & $72.9(15.3)$ & $77.7(13.2)$ & 11.24 & 11.61 (8.18-15.04) & $<.001$ \\
\hline
\end{tabular}

improve inhaler adherence and technique over usual care for patients with COPD. In this low-income, predominantly minority population living with moderate to severe COPD, 9 months of health coaching resulted in greater patient-reported adherence to inhaled controller medications and a threefold increase in correct observed inhaler technique.

Our health-coaching model is responsive to recent calls to incorporate attention to shared decision making and collaborative care into efforts to improve adherence to inhaled medications for COPD. ${ }^{36,59}$ In a systematic review of interventions to improve adherence to COPD controller medications, Bryant and colleagues ${ }^{36}$ observed that shared decision makingshown to improve medication adherence for other conditions, including asthma ${ }^{60}$ — was missing from the literature on interventions for COPD. Our study addresses this gap, as our intervention, health coaching, is grounded in the principles of shared decision making, for example, selection of devices based on patient capabilities and preferences.

Collaborative care models using unlicensed team members to assist patients with navigation or education have improved quality of care and adherence to treatment in populations with other complex conditions and comorbidities. ${ }^{35,61-63}$ Our model is aligned with a recently proposed model for COPD collaborative care, ${ }^{64}$ which includes as core tenets access, teamwork, disease management, and coordination of care. Health coaching addresses each of the pillars of this model, providing a point of contact to facilitate access; additional time from a new team member who plays a key role in activating the patient as a core member of the team (teamwork); proactive review of treatment plans and preventive care to improve disease management and active coordination of care among primary care, specialty care, and inpatient care. One key technique in health coaching that may have contributed to improved inhaler technique is closing the loop, ${ }^{50}$ or asking patients to demonstrate use of their inhalers and providing targeted feedback to improve use, which has been shown to improve technique in several small COPD studies. ${ }^{65,66}$ Use of closing the loop in practice is uncommon, with only $27 \%$ to $50 \%$ of patients reporting that their health care team has ever observed them use their inhalers. ${ }^{67,68}$ It is estimated that effective inhaler instruction using closing the loop requires approximately 5 minutes for devices such as the Diskus and 8 minutes for metered dose inhalers, ${ }^{69}$ time that may be more feasible in a collaborative model wherein health coaches share responsibility for patient care.

The direction of change for the primary outcomes in the AIR study, such as disease-specific quality of life and exacerbations, were positive for the coached group, but differences did not reach statistical significance over usual care. ${ }^{70}$ This result may suggest that improved inhaler technique and adherence are only one of the factors required to move outcomes.

\section{Limitations}

Our study was conducted in an urban, low-income population with moderate to severe COPD; generalizability of the intervention to other settings requires additional evaluation. The prevalence of people with COPD who had never smoked tobacco is consistent with that in other studies conducted in clinical settings $\mathrm{s}^{71,72}$ but lower than estimates for the general population. ${ }^{73}$ Loss to follow-up was greater in the health-coaching arm than in the usual-care arm but did not appear to differ by study arm for baseline characteristics. ${ }^{73}$ Adherence was self-reported. Like other studies of inhaler technique, our study is limited by a lack of standard definitions for critical errors and common checklists. ${ }^{32}$ Although inhaler adherence has been linked to improved outcomes in other studies, ${ }^{5,9,74}$ the AIR study was not able to demonstrate reduced 
exacerbations or improved quality of life despite better adherence with health coaching.

\section{Conclusions}

Patients who received 9 months of health coaching reported greater adherence to their COPD inhaled medications and were directly observed to use their inhalers correctly more often than patients receiving usual care. Given that COPD is a leading cause of hospital readmissions, health systems have a financial incentive to provide support for self-management of this disease, which may include improvement of inhaler use as a component strategy. Health coaching such as that provided in the AIR study may provide a scalable model to improve inhaler use for people living with COPD.

\section{To read or post commentaries in response to this article, see it online at http://www.AnnFamMed.org/content/18/1/5.}

Key words: health coaching; self-management; patient education; medication adherence; inhalers; inhaler technique; chronic obstructive pulmonary disease; chronic illness; shared decision making; practicebased research; primary care

Submitted December 16, 2018; submitted, revised, April 8, 2019; accepted May 5, 2019.

Funding support: This work was funded by the Patient-Centered Outcomes Research Institute (PCORI AD-1306-03900). Data gathering and analysis was supported by the NIH/NCRR Colorado CTSI (grant number UL1 RR025780) through use of Redcap software.

Disclaimer: This article's contents are the authors' sole responsibility and do not necessarily represent official PCORI or NIH views.

Previous presentation: This work was previously presented at the North American Primary Care Research Group Annual Conference; November 9-12, 2018; Chicago, Illinois.

Trial registration: Clinicaltrials.gov NCT02234284.

Acknowledgments: The authors thank the clinicians, leaders, and staff at our clinic partners for their participation and active support of the AIR study. The authors also thank Eula Lewis, RRT, CTTS, AE-C, and the Community Spirometry Program at the Zuckerberg San Francisco General Hospital and Trauma Center for all the time and effort spent training our team and for sharing their wealth of knowledge. Members of the authors' study advisory board provided invaluable advice, insight, and new ideas that substantially improved the study. In particular, people living with COPD served in key advisory roles, both on the advisory board and as members of the core study team. They participated in formation of research questions, took part in selection and training of health coaches, advised on study methods and instruments, and participated in the interpretation of results. The authors owe a particular debt to Devon Low, a patient research partner, for his many insights and contributions to the AIR study.

\section{References}

1. National Heart, Lung, and Blood Institute. Morbidity and Mortality: 2012 Chart Book on Cardiovascular, Lung, and Blood Diseases. Bethesda, MD: National Institutes of Health; 2012.
2. Jencks SF, Williams MV, Coleman EA. Rehospitalizations among patients in the Medicare fee-for-service program. N Engl J Med. 2009;360(14):1418-1428.

3. Global Initiative for Chronic Obstructive Lung Disease. Global strategy for the diagnosis, management, and prevention of chronic obstructive pulmonary disease (2017 Report). https://goldcopd.org/ gold-2017-global-strategy-diagnosis-management-prevention-copd/. Published 2017. Accessed Feb 8, 2018.

4. Simoni-Wastila L, Wei YJ, Qian J, et al. Association of chronic obstructive pulmonary disease maintenance medication adherence with all-cause hospitalization and spending in a Medicare population. Am J Geriatr Pharmacother. 2012;10(3):201-210.

5. Vestbo J, Anderson JA, Calverley PM, et al. Adherence to inhaled therapy, mortality and hospital admission in COPD. Thorax. 2009; 64(11):939-943

6. Decramer M, Molenberghs G, Liu D, et al; UPLIFT investigators. Premature discontinuation during the UPLIFT study. Respir Med. 2011;105(10):1523-1530.

7. Mäkelä MJ, Backer V, Hedegaard M, Larsson K. Adherence to inhaled therapies, health outcomes and costs in patients with asthma and COPD. Respir Med. 2013;107(10):1481-1490.

8. DiMatteo MR. Variations in patients' adherence to medical recommendations: a quantitative review of 50 years of research. Med Care. 2004;42(3):200-209.

9. Toy EL, Beaulieu NU, McHale JM, et al. Treatment of COPD: relationships between daily dosing frequency, adherence, resource use, and costs. Respir Med. 2011;105(3):435-441.

10. Mueller S, Wilke T, Bechtel B, Punekar YS, Mitzner K, Virchow JC. Non-persistence and non-adherence to long-acting COPD medication therapy: a retrospective cohort study based on a large German claims dataset. Respir Med. 2017;122:1-11.

11. Jung E, Pickard AS, Salmon JW, Bartle B, Lee TA. Medication adherence and persistence in the last year of life in COPD patients. Respir Med. 2009;103(4):525-534.

12. Krigsman K, Moen J, Nilsson JL, Ring L. Refill adherence by the elderly for asthma/chronic obstructive pulmonary disease drugs dispensed over a 10-year period. J Clin Pharm Ther. 2007;32(6): 603-611.

13. Franssen FM, Spruit MA, Wouters EF. Determinants of polypharmacy and compliance with GOLD guidelines in patients with chronic obstructive pulmonary disease. Int J Chron Obstruct Pulmon Dis. 2011;6:493-501.

14. Restrepo RD, Alvarez MT, Wittnebel LD, et al. Medication adherence issues in patients treated for COPD. Int J Chron Obstruct Pulmon Dis. 2008;3(3):371-384.

15. George J, Kong DC, Stewart K. Adherence to disease management programs in patients with COPD. Int J Chron Obstruct Pulmon Dis. 2007;2(3):253-262.

16. George J, Kong DC, Thoman R, Stewart K. Factors associated with medication nonadherence in patients with COPD. Chest. 2005; 128(5):3198-3204.

17. Cecere LM, Slatore CG, Uman JE, et al. Adherence to long-acting inhaled therapies among patients with chronic obstructive pulmonary disease (COPD). COPD. 2012;9(3):251-258.

18. VanderSchaaf K, Olson KL, Billups S, Hartsfield CL, Rice M. Selfreported inhaler use in patients with chronic obstructive pulmonary disease. Respir Med. 2010;104(1):99-106.

19. Khdour MR, Hawwa AF, Kidney JC, Smyth BM, McElnay JC. Potential risk factors for medication non-adherence in patients with chronic obstructive pulmonary disease (COPD). Eur J Clin Pharmacol. 2012;68(10):1365-1373.

20. Hayton C, Clark A, Olive S, et al. Barriers to pulmonary rehabilitation: characteristics that predict patient attendance and adherence. Respir Med. 2013;107(3):401-407. 
21. Bourbeau J, Bartlett SJ. Patient adherence in COPD. Thorax. 2008; 63(9):831-838.

22. Bosley CM, Corden ZM, Rees PJ, Cochrane GM. Psychological factors associated with use of home nebulized therapy for COPD. Eur Respir J. 1996;9(11):2346-2350.

23. George M. Adherence in asthma and COPD: new strategies for an old problem. Respir Care. 2018;63(6):818-831.

24. Sanchis J, Gich I, Pedersen S, Aerosol Drug Management Improvement Team (ADMIT). Systematic review of errors in inhaler use: has patient technique improved over time? Chest. 2016;150(2): 394-406.

25. Melani AS, Zanchetta D, Barbato N, et al; Associazione Italiana Pneumologi Ospedalieri Educational Group. Inhalation technique and variables associated with misuse of conventional metered-dose inhalers and newer dry powder inhalers in experienced adults. Ann Allergy Asthma Immunol. 2004;93(5):439-446.

26. Fink JB, Rubin BK. Problems with inhaler use: a call for improved clinician and patient education. Respir Care. 2005;50(10):1360-1374; discussion 1374-1375.

27. Baverstock M, Woodhall N, Maarman V. P94 Do healthcare professionals have sufficient knowledge of inhaler techniques in order to educate their patients effectively in their use? Thorax. 2010; 65(Suppl 4):A1117-A1118

28. Melzer AC, Ghassemieh BJ, Gillespie SE, et al. Patient characteristics associated with poor inhaler technique among a cohort of patients with COPD. Respir Med. 2017;123:124-130.

29. Oliveira PD, Menezes AM, Bertoldi AD, Wehrmeister FC, Macedo SE. Assessment of inhaler techniques employed by patients with respiratory diseases in southern Brazil: a population-based study. J Bras Pneumol. 2014;40(5):513-520.

30. Pothirat C, Chaiwong W, Phetsuk N, Pisalthanapuna S, Chetsadaphan N, Choomuang W. Evaluating inhaler use technique in COPD patients. Int J Chron Obstruct Pulmon Dis. 2015;10:1291-1298.

31. Omachi TA, Sarkar U, Yelin EH, Blanc PD, Katz PP. Lower health literacy is associated with poorer health status and outcomes in chronic obstructive pulmonary disease. J Gen Intern Med. 2013; 28(1):74-81.

32. Usmani OS, Lavorini F, Marshall J, et al. Critical inhaler errors in asthma and COPD: a systematic review of impact on health outcomes. Respir Res. 2018;19(1):10.

33. Eisner MD, Blanc PD, Omachi TA, et al. Socioeconomic status, race and COPD health outcomes. J Epidemiol Community Health. 2011; 65(1):26-34

34. Wong AW, Gan WQ, Burns J, Sin DD, van Eeden SF. Acute exacerbation of chronic obstructive pulmonary disease: influence of social factors in determining length of hospital stay and readmission rates. Can Respir J. 2008;15(7):361-364.

35. Viswanathan $M$, Golin $C E$, Jones $C D$, et al. Interventions to improve adherence to self-administered medications for chronic diseases in the United States: a systematic review. Ann Intern Med. 2012; 157(11):785-795.

36. Bryant J, McDonald VM, Boyes A, Sanson-Fisher R, Paul C, Melville J. Improving medication adherence in chronic obstructive pulmonary disease: a systematic review. Respir Res. 2013;14:109.

37. Rand CS. Patient adherence with COPD therapy. Eur Respir Rev. 2005;14(96):97-101

38. Thom DH, Wolf J, Gardner H, et al. A qualitative study of how health coaches support patients in making health-related decisions and behavioral changes. Ann Fam Med. 2016;14(6):509-516.

39. Bennett HD, Coleman EA, Parry C, Bodenheimer T, Chen EH. Health coaching for patients with chronic illness. Fam Pract Manag. 2010;17(5):24-29.

40. Ghorob A. Health coaching: teaching patients how to fish. Fam Pract Manag. 2013;20(3):40-42.
41. Benzo R, Vickers K, Novotny PJ, et al. Health coaching and chronic obstructive pulmonary disease rehospitalization: a randomized study. Am J Respir Crit Care Med. 2016;194(6):672-680.

42. Rice KL, Dewan N, Bloomfield HE, et al. Disease management program for chronic obstructive pulmonary disease: a randomized controlled trial. Am J Respir Crit Care Med. 2010;182(7):890-896.

43. Wood-Baker R, Reid D, Robinson A, Walters EH. Clinical trial of community nurse mentoring to improve self-management in patients with chronic obstructive pulmonary disease. Int J Chron Obstruct Pulmon Dis. 2012;7:407-413.

44. Thom DH, Willard-Grace R, Hessler D, et al. The impact of health coaching on medication adherence in patients with poorly controlled diabetes, hypertension, and/or hyperlipidemia: a randomized controlled trial. J Am Board Fam Med. 2015;28(1):38-45.

45. Willard-Grace R, Chen EH, Hessler D, et al. Health coaching by medical assistants to improve control of diabetes, hypertension, and hyperlipidemia in low-income patients: a randomized controlled trial. Ann Fam Med. 2015;13(2):130-138.

46. Fisher EB, Strunk RC, Highstein GR, et al. A randomized controlled evaluation of the effect of community health workers on hospitalization for asthma: the asthma coach. Arch Pediatr Adolesc Med. 2009;163(3):225-232

47. Thom DH, Willard-Grace R, Tsao S, et al. Randomized controlled trial of health coaching for vulnerable patients with chronic obstructive pulmonary disease. Ann Am Thorac Soc. 2018;15(10):1159-1168.

48. Huang B, Willard-Grace $R$, De Vore $D$, et al. Health coaching to improve self-management and quality of life for low income patients with chronic obstructive pulmonary disease (COPD): protocol for a randomized controlled trial. BMC Pulm Med. 2017;17(1):90.

49. University of California, San Francisco, Center for Excellence in Primary Care. Health Coaching Curriculum. http://cepc.ucsf.edu/content/health-coaching-curriculum. Published Sep 15, 2016. Accessed Mar 6, 2019

50. Bodenheimer T. Teach-Back: a simple technique to enhance patients' understanding. Fam Pract Manag. 2018;25(4):20-22.

51. Melani AS, Bonavia M, Cilenti V, et al; Gruppo Educazionale Associazione Italiana Pneumologi Ospedalieri. Inhaler mishandling remains common in real life and is associated with reduced disease control. Respir Med. 2011;105(6):930-938.

52. Melani AS, Canessa P, Coloretti I, et al; Educational Study Group of the Italian Association of Hospital Pulmonologists (AIPO). Inhaler mishandling is very common in patients with chronic airflow obstruction and long-term home nebuliser use. Respir Med. 2012; 106(5):668-676.

53. Fairclough DL. Design and Analysis of Quality of Life Studies in Clinical Trials: Interdisciplinary Statistics. Boca Raton, FL: Chapman \& Hall/ CRC; 2002.

54. Little RJA. Statistical Analysis With Missing Data. New York, NY: Wiley; 1987.

55. Kraemer HC, Wilson GT, Fairburn CG, Agras WS. Mediators and moderators of treatment effects in randomized clinical trials. Arch Gen Psychiatry. 2002;59(10):877-883.

56. Raudenbush SW, Bryk AS. Hierarchical Linear Models: Applications and Data Analysis Methods. 2nd ed. Thousand Oaks, CA: Sage Publications; 2002

57. Hedeker DR, Gibbons RD. Longitudinal Data Analysis. Hoboken, NJ: Wiley-Interscience; 2006

58. Vestbo J, Hurd SS, Agustí AG, et al. Global strategy for the diagnosis, management, and prevention of chronic obstructive pulmonary disease: GOLD executive summary. Am J Respir Crit Care Med. 2013;187(4):347-365.

59. Bender BG. Nonadherence in chronic obstructive pulmonary disease patients: what do we know and what should we do next? Curr Opin Pulm Med. 2014;20(2):132-137. 
60. Wilson SR, Strub P, Buist AS, et al; Better Outcomes of Asthma Treatment (BOAT) Study Group. Shared treatment decision making improves adherence and outcomes in poorly controlled asthma. Am J Respir Crit Care Med. 2010;181(6):566-577.

61. Bussing R, Zima BT, Mason DM, Meyer JM, White K, Garvan CW. ADHD knowledge, perceptions, and information sources: perspectives from a community sample of adolescents and their parents. J Adolesc Health. 2012;51(6):593-600.

62. Apter AJ, Wan F, Reisine $S$, et al. Feasibility, acceptability and preliminary effectiveness of patient advocates for improving asthma outcomes in adults. J Asthma. 2013;50(8):850-860.

63. Unutzer JHH, Schoenbaum M, Druss B. The Collaborative Care Model: An Approach for Integrating Physical and Mental Health Care in Medicaid Health Homes. Washington, DC: Health Home Information Resource Center; 2013.

64. Fromer L. Implementing chronic care for COPD: planned visits, care coordination, and patient empowerment for improved outcomes. Int J Chron Obstruct Pulmon Dis. 2011;6:605-614.

65. Press VG, Arora VM, Shah LM, et al. Teaching the use of respiratory inhalers to hospitalized patients with asthma or COPD: a randomized trial. J Gen Intern Med. 2012;27(10):1317-1325.

66. Dantic D. A critical review of the effectiveness of 'teach-back' technique in teaching COPD patients self-management using respiratory inhalers. Health Educ J. 2014;73(1):41-50.

67. Souza ML, Meneghini AC, Ferraz E, Vianna EO, Borges MC. Knowledge of and technique for using inhalation devices among asthma patients and COPD patients. J Bras Pneumol. 2009;35(9):824-831.
68. Dudvarski llic A, Zugic V, Zvezdin B, et al. Influence of inhaler tech nique on asthma and COPD control: a multicenter experience. Int J Chron Obstruct Pulmon Dis. 2016;11:2509-2517.

69. Melani AS, Bonavia M, Mastropasqua E, et al; Gruppo Educazionale Associazione Italiana Pneumologi Ospedalieri (AIPO). Time required to rectify inhaler errors among experienced subjects with faulty technique. Respir Care. 2017;62(4):409-414.

70. Thom DH, Willard-Grace R, Tsao S, et al. Randomized controlled trial of health coaching for vulnerable patients with chronic obstructive pulmonary disease. Ann Am Thorac Soc. 2018;15(10):1159-1168.

71. Barr RG, Celli BR, Mannino DM, et al. Comorbidities, patient knowledge, and disease management in a national sample of patients with COPD. Am J Med. 2009;122(4):348-355.

72. Coultas DB, Jackson BE, Russo R, et al. Home-based physical activity coaching, physical activity, and health care utilization in chronic obstructive pulmonary disease. Chronic obstructive pulmonary disease self-management activation research trial secondary outcomes. Ann Am Thorac Soc. 2018;15(4):470-478.

73. Salvi SS, Barnes PJ. Chronic obstructive pulmonary disease in nonsmokers. Lancet. 2009;374(9691):733-743.

74. Antoniu SA. Adherence to inhaled therapy in COPD: effects on survival and exacerbations. Expert Rev Pharmacoecon Outcomes Res. 2010;10(2):115-117.

\section{Get the Annals of} Family Medicine by E-mail

SEPTEMBER/OCTOBER 2018 ․ Vol 16, No. 5

Editorials | Original Research | Research Briefs I Systematic Reviews I Reflections I Innovations I Departments

\section{Editorial Fellowship: Now accepting applications}

The full text of the journal is available online at hittp://www. annfammed.org and through various gregators, including PubMed Central, EBSCO, and MDConsult. The Annals is indexed in the MEDLINE, Science Citation Index Expanded, Current Contents/Clinical Medicine, EMBASE, and CINHAL databases.

\section{EDITORIALS}

In This lssue: Nothing Simple Kurt C. Stange

\section{The Long Loneliness of Primary Carey} Timothy P. Daaleman

Clinical Prediction Rules: Challenges, Barriers, and Promises Emma Wallace; Michael E. Johansen

\section{ORIGINAL RESEARCH}

Social lsolation and Patient Experience in Older Adults

Takuya Aoki; Yosuke Yamamoto; Tatsuyoshi Ikenoue; Yuka Urushibara-Miyachi; Morito Kise; Yasuki Fujinuma; Shunichi Fukuhara

Social isolation is associated with a negative patient experience in older primary care patients in Japan. 